\title{
Processo de Desenvolvimento do Sistema Multi-Agentes Monitor Glicêmico
}

\author{
D. G. Tavares ${ }^{1}$, J. Eichler ${ }^{1}$, L. F. Pereira ${ }^{1}$, T. S. Silva, A. L. F. da Cunha ${ }^{1}$, \\ R. F. A. Martins ${ }^{1}$, L.M. Cysneiros ${ }^{2}$, V. M. B. Werneck ${ }^{1}$ \\ ${ }^{1}$ UERJ - Universidade Estadual do Rio de Janeiro \\ Departamento de Informática e Ciência da Computação \\ Rio de Janeiro - Brasil \\ ${ }^{2}$ York University - School of Information Technology, \\ Toronto - Canada
}

vera@ime.uerj.br, cysneiro@yorku.ca

\begin{abstract}
Resumo
O paradigma orientado a agentes introduz conceitos como proatividade e autonomia para obter sistemas mais flexíveis e robustos em ambientes computacionais complexos. Sistemas Multi-Agentes provêem a representação e implementação de novas aplicações com requisitos e características das organizações sociais, como autonomia, proatividade e mobilidade. $O$ tratamento de diabetes requer um controle diário e acompanhamento sistemático do paciente, necessitando muitas vezes de ajustes perante as situações do dia a dia. Assim este trabalho descreve o processo de desenvolvimento de um Sistema MultiAgentes para Controle de Diabetes centrado no paciente que, em situações críticas, emite alertas. Este trabalho apresenta uma visão geral do sistema, sua modelagem e implementação na web e em dispositivos móveis.
\end{abstract}

\begin{abstract}
The agent-oriented approach to software engineering introduces concepts such as proactiveness and autonomy to achieve more flexible and robust systems for complex application environments. The diabetes treatment requires daily monitoring of the patient, systematic follow-up by the physician and in many cases adjustments according to day by day variations. Therefore this work describes the software process of the Glycemic Monitor System, a MultiAgents System to Control Diabetes, focused on the patient, setting up alerts in critical situations. This paper presents an overview of this system, its modeling and development in a web and in a mobile plataform.
\end{abstract}

\section{Introdução}

O paradigma orientado a agentes para desenvolvimento de software tem se destacado como uma abordagem para tratar requisitos que vêm surgindo com a grande proliferação de componentes de software distribuídos por grande número de usuários em distribuições geográficas que podem atingir escala mundial. Necessidades proeminentes dessas novas aplicações, como autonomia e sociabilidade não são tratadas pelos atuais paradigmas. No contexto emergente, uma das principais características destes componentes de software (visto aqui como agentes) é ter autonomia para decidir se um serviço requisitado por outro agente será ou não realizado em tempo real. Esta decisão é baseada nas necessidades individuais de quem estiver usando esse agente. Em decorrência dessa autonomia, aspectos de sociabilidade como a dependência de um agente em relação a outro e quão crítica essa dependência é devem ser tratados desde o principio dos processos de desenvolvimento de software.

Este trabalho tem como objetivo apresentar o Sistema Multi-Agentes de Apoio a Diabéticos denominado Monitor Glicêmico e seu processo de desenvolvimento. Este sistema foi definido com base no Guardian Angel [Szolovits et al, 1994] e o especializa detalhando para que pacientes com diabetes possam acompanhar seu progresso e ter seu tratamento continuamente avaliado (especializa detalhando???). Monitor Glicêmico foi construído num ambiente com diversos agentes que rodam em diferentes ambientes.

Neste artigo descrevemos o processo de desenvolvimento do Sistema Multi-Agentes Monitor Glicêmico, sua modelagem e seus protótipos. Na seção 2 daremos uma visão geral do sistema. Na seção 3 descreveremos o processo de desenvolvimento utilizado e sua modelagem. Na seção 4 apresentaremos o protótipo desenvolvido em PDA e na seção 5 destacaremos alguns trabalhos correlatos, nossas conclusões e sugestões para trabalhos futuros.

\section{Visão geral do Monitor Glicêmico}

O sistema Monitor Glicêmico surgiu de uma especialização do Guardian Angel [Szolovits et al, 
1994] para o tratamento de pacientes com Diabetes Mellitus.

Guardian Angel é uma proposta de sistema de apoio ao tratamento médico, que permite ao paciente um controle maior sobre as suas informações de saúde e progressos dos tratamentos. A idéia é se ter um sistema voltado para o paciente onde este possa ter auxilio integral desde $o$ tratamento até às informações financeiras e médicas, voltado principalmente para doenças crônicas, tais como diabetes, pressão alta e esquizofrenia.

Monitor Glicêmico foi construído baseado, também, no conhecimento existente e disponível nos sites da Sociedade Brasileira de Diabetes (2008), da Federação Internacional de Diabetes (2008) e da Associação Americana de Diabetes (2008). Um paciente diabético precisa de contato constante com um médico para acompanhar o seu tratamento. Com a idéia do Guardian Angel instanciada no Monitor Glicêmico, é possível fazer isto, sem sair de casa ou marcar consulta. O sistema permite monitorar taxas de açúcar através de um medidor portátil e registrá-las no computador.

Para isso, o sistema é composto de um grupo de agentes de softwares chamados de "anjos guardiães" (GA), integrando todas as considerações necessárias para a saúde do paciente, incluindo considerações médicas legais e informações financeiras. Este sistema pessoal irá auxiliar no acompanhamento, gerência e interpretação do histórico de saúde do paciente, assim como fornecer recomendações tanto para o paciente como para o prestador de serviço. O sistema tem também a incumbência de manter, de forma reduzida, compreensiva, acumulativa, correta e coerente os registros médicos, de forma que estejam rapidamente acessíveis, independentemente de época e provedor de serviço.

Cada GA é um processo ativo que executa várias funções importantes: coleta de dados do paciente; verificação, interpretação e explicação dos fatos relevantes ou planos médicos; adequação de suas recomendações às experiências adquiridas e preferências do paciente; realização de avaliação de viabilidade, quanto à eficácia médica e o custo dos diagnósticos e planejamentos terapêuticos; monitoramento dos progressos da saúde do paciente; comunicação com outros softwares agentes, dos prestadores de serviço; auxilio na educação, informação e apoio ao paciente. Todas estas facilidades contribuem para a melhoria na qualidade do diagnóstico médico, aumentando o comprometimento do paciente e reduzindo os efeitos da doença e os erros médicos.

\section{Processo de desenvolvimento do Monitor Glicêmico}

O processo de desenvolvimento do Sistema Monitor Glicêmico utilizou o modelo de processo de desenvolvimento de sistemas multi-agentes proposto pelo método MAS-Common-KADS [Iglesias e Garijo, 2005]. Este método foi escolhido devido ao conhecimento especialista do Sistema Monitor Glicêmico, pois esse método é uma extensão do método CommonKADS [Schreiber et al, 1999]. CommonKADS tornou-se, principalmente na Europa, uma referência no desenvolvimento de sistemas baseados em conhecimento (SBC).

$\mathrm{O}$ modelo de processo do MAS-Common-KADS engloba as seguintes fases: Conceituação, Análise, Design, Codificação, Integração, Operação e Manutenção. A fase de Conceituação consiste na tarefa de elicitação para obter uma primeira descrição do problema e a determinação dos casos de uso que podem ajudar a entender os requisitos informais e a testar o sistema. A Análise determina os requisitos do sistema partindo do enunciado do problema. Durante esta fase se desenvolvem os seguintes modelos: organização, tarefas, agentes, comunicação, coordenação e experiência. No Design define-se como os requisitos da fase de análise podem ser conseguidos mediante o desenvolvimento do modelo de design, determinando-se as arquiteturas tanto da rede multiagentes como de cada agente. Na Codificação cada agente é implementado e testado e na fase de Integração, o sistema completo é testado.

MAS-CommonKADS [Iglesias, 1998], [Iglesias e Garijo, 1999], [Iglesias e Garijo, 2005] é uma extensão da metodologia CommonKADS englobando aspectos que são relevantes para sistemas multi-agentes. CommonKADS tem o Modelo de Experiência como centro da metodologia, com objetivo de modelar o conhecimento heurístico de resolução de problemas empregado por um agente para realizar uma tarefa. A proposta MAS-CommonKADS é centrada no Modelo de Agentes.

O processo de identificação dos requisitos do sistema Monitor Glicêmico foi realizado nas fases de Conceituação e Análise seguida de uma fase de Design e codificação dos protótipos. Foram definidos sete modelos: Modelo de Agentes, Modelo de Organização, Modelo de Tarefas, Modelo de Experiência, Modelo de Comunicação, Modelo de Coordenação e Modelo de Design. A descrição completa da modelagem e codificação pode ser encontrada em [Almentero e van Boekel, 2007], [Cunha e Martins, 2007], [Pereira e Silva, 2005] e [Tavares e Werneck, 2009].

\subsection{Fase de Conceituação}

O objetivo desta fase é obter uma primeira descrição do problema e do sistema que será desenvolvido. O documento desta fase teve como objetivo modelar um protótipo do Monitor Glicêmico, implementando algumas das funções propostas inicialmente ao sistema. Nessa fase de conceituação foram realizadas as seguintes atividades: descrição do problema, identificação dos atores, identificação e descrição dos casos de uso, definição dos diagramas de casos de uso e dos diagramas de seqüência de mensagens. 
Os seguintes atores foram identificados no sistema: o paciente, o médico e o responsável pelo paciente. Nesta primeira versão, o Paciente é uma Pessoa com diabetes mellitus em terapia insulínica intensiva, com múltiplas doses. O paciente acessará o sistema com o objetivo de auxiliar a administração de alguns aspectos do tratamento. O Médico é responsável pelo tratamento do paciente usuário do sistema e deverá ter completo conhecimento sobre as informações contidas no sistema e sobre o papel deste no tratamento. Ocasionalmente, o médico receberá mensagens do sistema informando a ocorrência de desvios de padrão na taxa glicêmica do paciente. No futuro esses alertas poderão ser configurados pelo médico para que este se sinta confortável com o nível de alerta sem ser incomodado muitas vezes por dia. O Responsável pelo acompanhamento do tratamento do paciente tem o papel de acompanhar o andamento do tratamento, recebendo alertas informando sobre desvios graves no padrão da taxa glicêmica. Todas as funcionalidades relacionadas aos três atores podem rodar em PDA ou telefones celulares, este último, principalmente, no caso de emergências.

Para cada ator foram identificados vários casos de uso. Por exemplo, no caso de uso UC06-Enviar Alertas, o paciente participa recebendo alertas de nível leve, médio e grave, enquanto o médico recebe alertas de nível médio e grave, e o responsável somente alertas de nível grave. Na descrição dos casos de uso foram descritos os fluxos de eventos de cada um dos casos de uso identificados e estes fazem referência aos agentes que constituem o sistema. Esses agentes foram descritos de maneira detalhada no modelo de agentes. Os Diagramas de Sequiência de Mensagens formalizam as interações e usam a notação do diagrama de sequiência de mensagens do UML.

\subsection{Fase de Análise}

Nesta fase foram desenvolvidos os Modelos de Agentes, de Tarefas, de Conhecimento, de Organização, de Coordenação e de Comunicação.

$\mathrm{O}$ modelo de agentes tem o objetivo de descrever os agentes que participam da solução dos problemas e são descritos nas Planilhas de CRC (ClassesResponsabilidades-Colaborações), nos diagramas de casos de uso interno (extensão do conceito de casos de uso do UML para agentes humanos e de software), nos diagramas de seqüência de mensagens relativas aos casos de uso internos, nas Planilhas de Agentes e na Tabela de Distribuição de Tarefas e Agentes. As atividades realizadas na modelagem de agentes foram a identificação dos agentes, a descrição dos agentes, a classificação dos agentes, a identificação, diagramação e descrição dos casos de uso interno e seus respectivos diagramas de seqüência de mensagens. Os diagramas de caso de uso interno descrevem as funções desempenhadas internamente pelos agentes.

A partir da fase de conceituação, foi possível identificar os Agentes Humanos: Paciente, Médico e Responsável; e os Agentes de software: Gerenciador de Dados Pessoais, Gerenciador de Alimentação, Gerenciador de Alertas, Gerenciador de Insulina, Gerenciador de Exercícios, Gerenciador do Banco de Dados e Avaliador de Tratamento.

Os agentes foram agrupados por categorias, observando as semelhanças e diferenças entre cada agente. Assim pudemos, por exemplo, ter uma Classe de Agentes Especialistas que representa os agentes que utilizam conhecimentos de um especialista na área de diabetes para produzir informações úteis ao paciente. Os Agentes classificados nessa categoria são: Gerenciador de Insulina, Gerenciador de Exercícios e Avaliador de Tratamento.

As planilhas CRC proporcionam um método para organizar as classes facilitando a aplicação dos conceitos de orientação a agentes. Para cada agente de software foi construída uma planilha CRC como apresentado para agente Avaliador de Tratamento na Tabela 1.

No sistema Monitor Glicêmico foram identificados vários casos de uso internos, sendo que a Figura 1 ilustra um exemplo de Diagrama de Casos de Uso Interno. As Descrições dos Casos de Uso Internos foram descritas de forma idêntica aos casos de uso através dos fluxos de eventos que fazem referência aos agentes que constituem o sistema.

Tabela 1. Planilha CRC para o Avaliador de Tratamento.

\begin{tabular}{|l|l|l|c|}
\hline \multicolumn{1}{|c|}{ Agente: Avaliador de Tratamento } & \multicolumn{1}{|c|}{ Plasse: Especialistas } \\
\hline \multicolumn{1}{|c|}{ Objo } & \multicolumn{1}{c|}{ Conhecimento } & \multicolumn{1}{c|}{ Colaborador } \\
\hline $\begin{array}{l}\text { Cadastrar taxa de glicose, dose de } \\
\text { insulina e resultado da avaliação. }\end{array}$ & $\begin{array}{l}\text { Solicitar cadastro ao Gerenciador do } \\
\text { Banco de Dados. }\end{array}$ & $\begin{array}{l}\text { Formato da avaliação e da taxa de } \\
\text { glicose. }\end{array}$ & $\begin{array}{c}\text { Gerenciador do Banco de } \\
\text { Dados. }\end{array}$ \\
\hline $\begin{array}{l}\text { Verificar se os resultados do } \\
\text { tratamento estão dentro dos padrões } \\
\text { esperados. }\end{array}$ & $\begin{array}{l}\text { Comparar resultados atuais com o } \\
\text { esperado e solicitar ao Gerenciador } \\
\text { de alertas o envio de alertas. }\end{array}$ & $\begin{array}{l}\text { Formato dos alertas, níveis normais } \\
\text { de taxa glicêmica e da dose de } \\
\text { insulina, histórico de utilização de } \\
\text { insulina, histórico da taxa glicêmica. }\end{array}$ & Gerenciador de Alertas. \\
\hline
\end{tabular}

Os diagramas de seqüência de mensagens apresentam a interação entre os agentes, conforme exemplifica a Figura 2. Ainda no Modelo de Agentes foram construídas as planilhas de agentes cujo objetivo é descrever melhor as capacidades e conhecimentos dos agentes. 
O Modelo de Tarefas é outro modelo elaborado na fase de Análise e permite mostrar a decomposição funcional do sistema. A descrição das tarefas se completou na identificação e descrição de seus objetivos. Esse detalhamento contém os parâmetros de entrada e saída, as condições de ativação e finalização além da descrição do processamento e tipo de objetivo. Para finalizar o Modelo de Tarefas e de Agentes foi elaborada uma Tabela com a Distribuição das Tarefas e dos Agentes onde essas tarefas são descritas nos casos de uso interno.
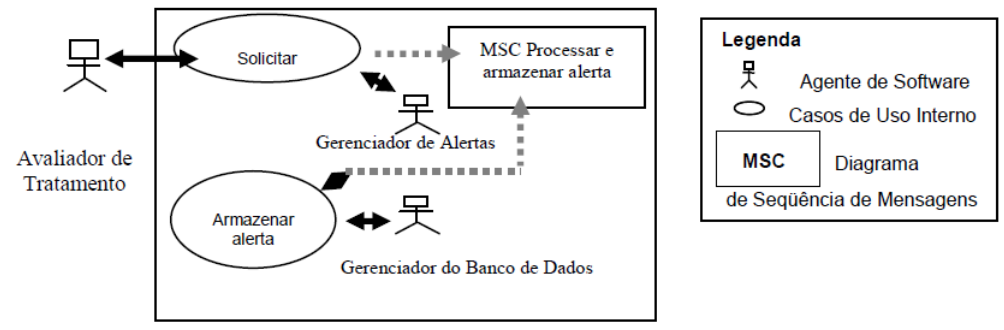

Figura 1. Diagrama de Casos de Uso Interno UC06 - Enviar Alerta

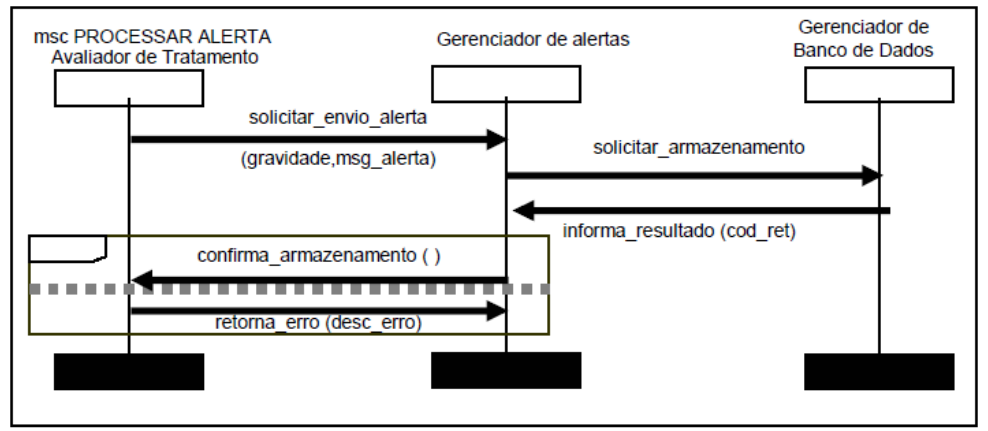

Figura 2. Diagrama de Seqüência de Mensagens: Processar e armazenar alerta.

O Modelo de Conhecimento foi, também, definido na fase de Análise, sendo composto das Estruturas de Domínio, Inferências e de Tarefas. Neste sistema foram identificados conceitos agrupados nos seguintes esquemas do domínio: Esquema-paciente, Esquemainsulina e Esquema-exercício. A Estrutura de Inferência foi especificada para os agentes inteligentes mediante a definição das inferências que são realizadas para a resolução do problema e da estrutura que relaciona as inferências e os papéis do conhecimento. Essa estrutura foi definida e instanciada para o domínio do problema a partir de estruturas genéricas contidas numa biblioteca de modelo [Schreiber et al, 1999]. A Estrutura de Tarefas representa de forma procedural as tarefas primitivas definidas da estrutura de inferência. No Monitor Glicêmico especificamos a Estrutura de Inferência e de Tarefas para o Avaliador de Tratamento.

O Modelo de Organização construído permitiu analisar as relações estruturais entre os agentes, tanto de software quanto humanos, que interagem com o sistema. Finalmente o último modelo elaborado na fase de Análise foi o Modelo de Coordenação cujo principal objetivo foi definir as interações entre agentes, permitindo um estudo mais aprofundado das interações homem-máquina e máquina-máquina. Para cada interação foi definido um Diagrama de Fluxo de Eventos, descrevendo como os agentes processam as mensagens recebidas e como enviam a mensagem através dos Diagramas de Transição de Estado na notação do UML [Rumbaugh, Jacobson e Booch, 1999].

\subsection{Fase de Design e de Codificação}

Nesta fase os componentes foram identificados e descritos gerando os seguintes artefatos: (i) infraestrutura de redes dos agentes, (ii) plataforma de implementação e (iii) projeto dos agentes.

A rede dos agentes do Monitor Glicêmico em diferentes plataformas sugere a existência de uma rede GA_NET, composta basicamente pelos ambientes GA_Authentication, GA_Hospital, GA_Clinic, GA_Physician, GA_Home e GA_PDA. O GA Authentication Server, é o servidor onde existe a aplicação responsável pela segurança do ambiente, o GA_Hospital é a entidade que se comunica com o hospital, o GA_Clinic existirá em cada clínica pertencente à rede, o GA_Physician existirá em cada consultório médico associado à rede, o GA_Home existirá na estação da residência de cada paciente em tratamento e o GA_PDA é utilizado individualmente pelo próprio paciente. Na Figura 3 temos uma visão geral do GA_NET e a relação entre os vários ambientes

do

GA. 


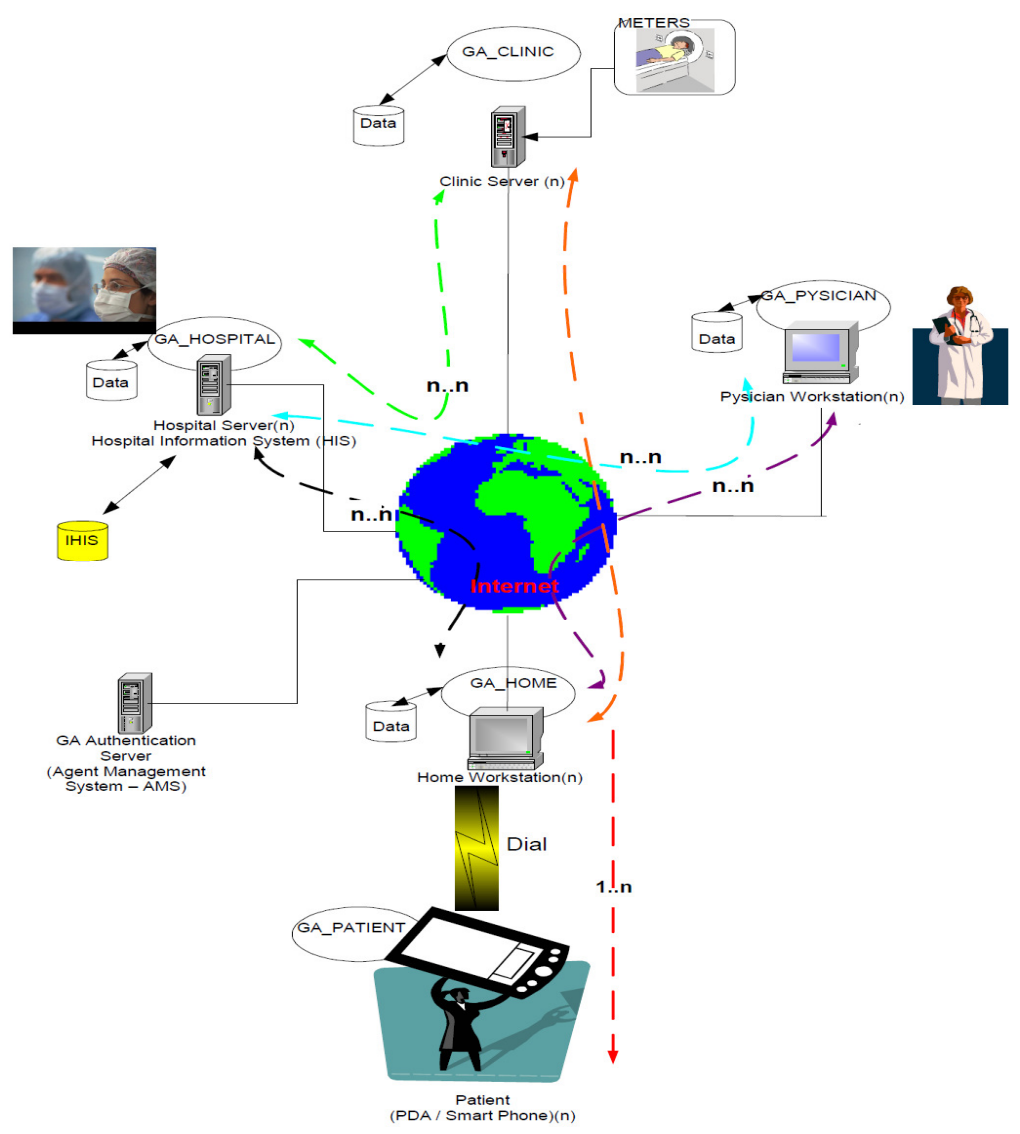

Figura 3. Visão Geral do Monitor Glicêmico baseado em um grupo de agentes de softwares (GA)

Devido a complexidade do sistema Monitor Glicêmico o projeto detalhado considerou apensas os agentes nos ambiente do GA-Home e GA-Patient e foram definidas duas plataformas Web e PDA (dispositivos móveis), para permitir o acesso de qualquer lugar via Internet. Além de outras vantagens, estas plataformas eliminam as etapas de instalação e configuração do software o que facilita o primeiro contato com o usuário.

Os agentes, Gerenciador de Dados Pessoais, Gerenciador de Alimentação, Gerenciador de Alertas, Gerenciador de Insulina, Gerenciador de Exercícios, Gerenciador do Banco de Dados e Avaliador de Tratamento foram projetados para implementação em Java segundo um padrão textual disponível no MASCommonKADS.

A primeira versão do ambiente utilizado para o GAHome foi Java, JSP, HTML, JavaScript. e SQL e depois alguns agentes foram implementados em JADE (2006). E a versão para PDA foi desenvolvida com uso da API de Java Micro Edition (J2ME) (2008), tendo a necessidade de redefinir a interface com o usuário e otimizar alguns algoritmos.

\section{Protótipos do Monitor Glicêmico}

Nessa seção daremos uma visão geral dos protótipos construídos com base nas modelagens definidas na seção 4. O acesso do sistema para o Ga-Home é realizado através de um $\operatorname{login}^{1}$ e uma senha disponibilizando suas funções. Este protótipo foi testado a nível operacional, sendo identificados alguns pontos que devem ser melhorados, sendo que destacamos principalmente as regras de inferência, pois o conhecimento foi retirado de livros e de sites [SBD, 2007] [ADA, 2007], [IDF, 207] sem uso de especialistas. A Figura 4 ilustra uma interação do sistema mostrando o cálculo da dosagem de insulina e o resultado da avaliação do paciente.

Através do Monitor Glicêmico, o paciente pode acompanhar seu progresso e ter seu tratamento continuamente avaliado de forma independente, ou seja, o diabético pode realizar consultas básicas - como calcular a taxa de carboidratos consumida em uma refeição - sem haver necessidade de consultar um médico.

A versão do Monitor Glicêmico para PDA foi construída com base nos documentos e códigos da versão para Web onde foram desenvolvidos os agentes: Gerenciador de Alimentação, Gerenciador de Insulina, Gerenciador de Exercício e Avaliador de Tratamento e,

\footnotetext{
1 O sistema pode ser acessado para teste com Usuário: vera http://152.92.106.36:8080/GAngel/paginas/html/login.jsp
} 
por último, Gerenciador de Banco de Dados. Essa versão para PDA foi desenvolvida para verificar a potencialidade do PDA para esse sistema, a adequabilidade da modelagem e simular a comunicação entre os agentes. Entretanto, a comunicação dos agentes foi simplificada e foi verificado que a modelagem só necessitou ser adaptada no caso do Modelo de Coordenação e o agente Gerenciador de Banco de Dados teve que ser reprojetado e codificado com algoritmos diferentes que otimizassem o acesso devido a restrições de memória e da diferença da operabilidade da interface do PDA.

O Agente Gerenciador de Alimentação no PDA consiste em uma lista que informa algumas informações nutricionais sobre os alimentos cadastrados, como a quantidade de carboidratos - em gramas - e calorias - em kcal. Na lista há 540 alimentos, porém o sistema possibilita que o paciente adicione ou remova os pratos que desejar. Para se adicionar um alimento, deve-se informar o nome deste, a medida na qual as informações se baseiam, e os valores nutricionais correspondentes, como mostra a Figura 5.a.

O Agente Gerenciador de Insulina é responsável pelo Cálculo da dosagem de insulina permitindo que o diabético calcule a dose de insulina que deve ser injetada para alcançar a taxa ideal de açúcar no sangue. Para isso, o paciente informa ao sistema o valor de sua taxa de glicose antes da alimentação, e seleciona os alimentos que deseja consumir em uma determinada refeição. A partir desses dados, o sistema fornece o perfil glicêmico atual do diabético, indicando se o prato escolhido pode ou não prejudicar seu estado de saúde. Com isso, o paciente pode optar por outro prato e refazer os cálculos para, então, se alimentar sem comprometer o organismo.

Os resultados deste cálculo ficam armazenados em um histórico para que tanto o paciente quanto o médico possam acompanhar o progresso temporal do tratamento. A Figura 5.b mostra um exemplo desse cálculo. Este agente ao calcular a dose de insulina solicita o Avaliador de Tratamento que analise a situação do paciente com os dados atuais e seu histórico nos últimos três dias e emite um alerta ao Agente Gerenciador de Alerta que deve tomar as providências necessárias.

\section{Conclusões}

O desenvolvimento de sistemas multi-agentes (MAS) e sistemas médicos não é trivial e necessita de uma atenção especial na sua engenharia de software para apoiar a definição de todos os requisitos de um sistema que trabalha com conhecimento e comunicação entre agentes. Assim, procuramos destacar neste trabalho a importância de se ter um processo de desenvolvimento de MAS que assegura a realização das atividades, apoiado a uma estratégia de definição dos requisitos e sua implementação.

Neste contexto foi proposto e apresentado o sistema multi-agentes Monitor Glicêmico que, através de um processo sistemático de desenvolvimento com método MAS-Common-KADS, modela e implementa os requisitos em diferentes plataformas.

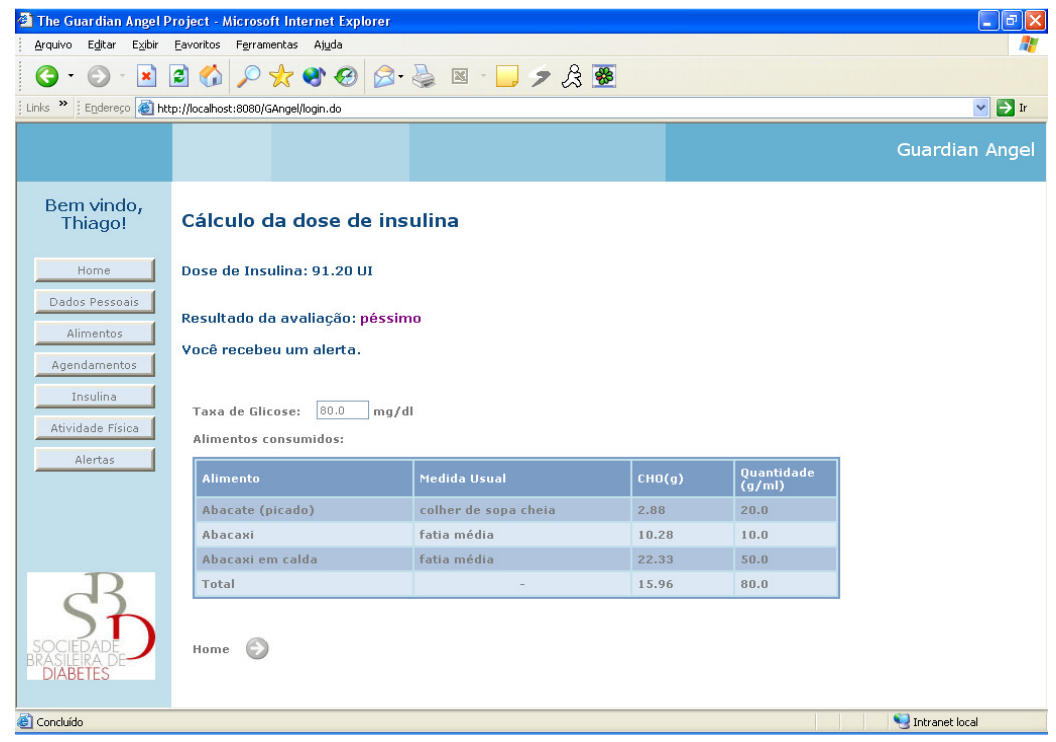

Figura 4. Monitor Glicêmico - Resultado do cálculo da dose de insulina 


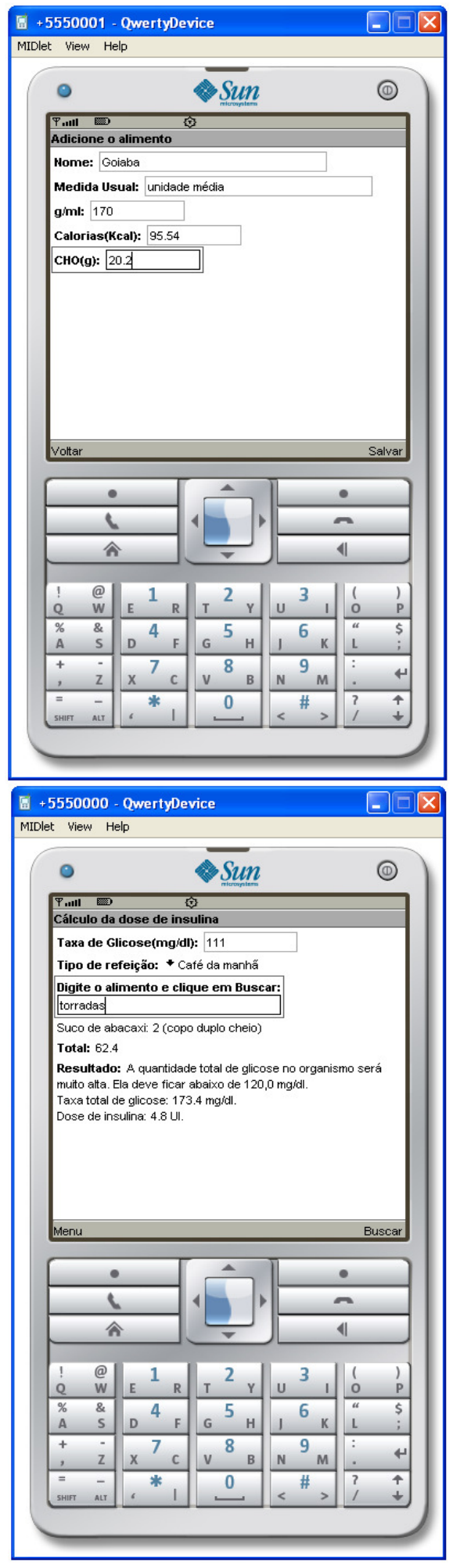

Figura 5: Monitor Glicêmico em PDA com as seguintes telas: a) Banco de alimento b) Resultado do cálculo da dose de insulina

Kirkman, Williams, Cafrey e Manrrero (2002) destacam a dificuldade de se obter melhorias no tratamento da diabetes utilizando estruturas tradicionais centradas nos médicos. Eles propõem um suporte nutricional computadorizado auxiliando o paciente no planejamento de cardápios de refeições. $\mathrm{Na}$ literatura MAS encontramos algumas propostas de desenvolvimento de sistemas multi-agentes para área de saúde tais como projeto IM-Agents [Mabry, Hug e Roundy, 2004], TeleDM [Ito, 2009]. Entretanto, nenhum desses sistemas utiliza um processo e uma metodologia para o desenvolvimento do sistema multiagentes.

A continuação deste trabalho consistirá na melhoria da base de conhecimentos utilizando especialistas para apoiar todo o processo de validação com objetivo de melhorar o desempenho em suas respostas e uso na prática do modulo PDA. O uso de uma linguagem baseada em lógica para comunicação entre os agentes é outro aspecto que pode ser incorporado ao sistema para permitir um melhor desempenho.

\section{Referências}

Almentero, E. K. e van Boekel R. O. (2007). Uma Avaliação dos Frameworks JADE e OpenCybele, Trabalho de Conclusão de Curso, Universidade do Estado do Rio de Janeiro.

ADA, American Diabetes Association. Disponível em. www.diabetes.org., acessado em maio 2007.

Cunha, A. L. F. da e Martins, R. F. A. (2007), 'Um comparativo entre as propostas de modelagem de sistemas usando Orientação a Agentes - AUML e MaSE: Modelagem do Guardian Angel”, Trabalho de Conclusão de Curso. (Graduação em Informática) - Universidade do Estado do Rio de Janeiro.

IDF, International Diabetes Federation. Disponível em. http://www.idf.org/e-atlas, acessado em maio 2007.

Iglesias, C. Á. (1998) "Definición de una Metodología para el Desarrollo de Sistemas Multiagente", Tese de Doutorado, Departamento de Engenharia de Sistemas de Telecomunicação, Universidade Politécnica de Madri. Madri, 322p.

Iglesias, C.A. e Garijo, M. (1999) "UER Technique: Conceptualisation for Agent-Oriented Development", In Proceeding of 5th International Conference on Information Systems Analysis and Synthesis (ISAS'99), Vol 5, p. 535-540.

Iglesias, C.A. e Garijo, M. (2005) “The Agent Oriented Methodology MAS-CommonKADS; IN AgentedOriented Methodologies", B. Henderson-Sellers e P. Giorgini (ed.), IDEA Group Publishing, p. 46-78.

Ito, M.; Silva, L. R.; Martini, J. ; Iochida, L., "Um Sistema Multi-agente para a Monitoração de Pacientes Diabéticos com Base no Modelo GRPC", In: IX Workshop de Informática Médica, 2009, Bento Gonçalves. Anais do XXIX Congresso da Sociedade Brasileira de Computação, 2009. p. 1935-1944.

JADE Java Agent Development Framework, http://jade.tilab.com/, acessado em junho de 2006.

Java, $\quad \underline{\text { http://java.sun.com/javame/reference/apis/jsr118/ }}$ acessado em abril 2008 
Kirkman, M.S., Williams, S.R., Cafrey H.H. e Manrrero, D.G. (2002), "Impact of Program to Improve Adherence to Diabetes Guidelines by Primary Care Physicians", Diabetes Care, v. 25, n. 11, p 1946-1951.

Pereira, L. e Silva, T. S. (2005), "Monitor Glicêmico: Um Sistema de Apoio a Diabetes. 2005". Trabalho de Conclusão de Curso. (Graduação em Informática) Universidade do Estado do Rio de Janeiro.

Mabry, S.L, Hug, C.R. Roundy, R.C.(2004), "Clinical Decision Support with IM-Agents and ERMA Multiagents", In: IEEE Symposium on Computer-Based Medical Systems, p. 242-247

Rumbaugh, J., Jacobson, I. e Booch, G. (1999) "The Unified Modeling Language Reference Manual"; AddisonWesley

SBD, Sociedade Brasileira de Diabetes. Disponível em. http://www.diabetes.org.br/. acessado em maio 2007.

Schreiber, G et al (1999), Knowledge Engineering and Management: The CommonKADS Methodology, Cambridge, MIT Press.

Szolovits, P., Doyle, J., Long, W.J., Kohane, I. e Pauker, S. G. (1994) "Guardian Angel: Patient-Centered Health Information Systems", Technical Report MIT/LCS/TR604.

Disponível

em. http://groups.csail.mit.edu/medg/projects/ga/manifesto/G Atr.html

Tavares, D. G. e Werneck, V. M. B. (2009), Monitor Glicêmico para PDA. In: $18^{a}$ SEMIC - Semana de Iniciação Científica, 2009, Rio de Janeiro. $20^{\mathrm{a}}$ UERJ Sem Muros 